\title{
Lightweight Infrared Sensing for Relative Navigation of Quadrotors
}

\author{
Mark Cutler, ${ }^{\star}$ Bernard Michini, ${ }^{\star}$ and Jonathan P. How ${ }^{\star \star}$ \\ Aerospace Controls Laboratory \\ Massachusetts Institute of Technology
}

\begin{abstract}
A lightweight solution for estimating position and velocity relative to a known marker is presented. The marker consists of three infrared (IR) LEDs in a fixed pattern. Using an IR camera with a $100 \mathrm{~Hz}$ update rate, the range and bearing to the marker are calculated. This information is then fused with inertial sensor information to produce state estimates at $1 \mathrm{kHz}$ using a sigma point Kalman filter. The computation takes place on a 14 gram custom autopilot, yielding a lightweight system for generating high-rate relative state information. The estimation scheme is compared to data recorded with a motion capture system.
\end{abstract}

\section{INTRODUCTION}

During the past decade significant advances have been made in the area of autonomous control and navigation of robotic systems, especially with regard to small, aerial vehicles navigating indoors where GPS signals are weak and often unusable [1,2]. Using a variety of sensors such as cameras, laser scanners, sonar, etc., aerial vehicles are now able to autonomously map and explore an unknown environment without the aid of GPS [3,4]. Generally, these estimation algorithms are concerned with estimating the state of the vehicle relative to the world within which it is operating.

Additionally, substantial research focus has been placed on controlling groups or flocks of autonomous agents. Inspired by observing flocking behaviors in species such as ants and bees, the idea is to create many small, relatively simple units that work together and collectively perform tasks impossible for any single agent to perform [5]. Vehicle state estimation and control in the context of robotic flocks is inherently different from the problems faced by a single vehicle exploring an unknown environment. Especially when

\footnotetext{
${ }^{\star}$ Ph.D. Candidates, Aerospace Controls Laboratory, MIT, cutlerm[at]mit. edu and bmich [at]mit.edu

${ }^{\star \star}$ Richard C. Maclaurin Professor of Aeronautics and Astronautics, Aerospace Controls Laboratory, MIT, jhow [at ] mit.edu
}

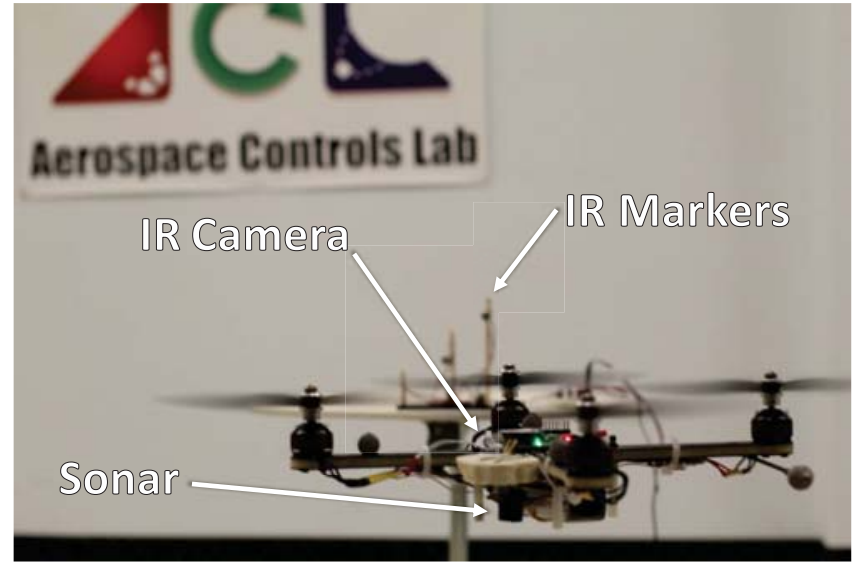

Fig. 1. Quadrotor used in experiments. The sonar faces down for measuring altitude and the IR camera faces forward for detecting range and bearing to the IR markers.

the agents are operating in close proximity to each other, each agent in the flock is concerned more with navigation relative to its neighboring agents than navigation and estimation relative to the world in which the flock is operating. The flock as a whole must still observe some notion of global state estimation and control; however, for the agents to navigate properly, global state information need not be available to every agent. The vast majority of the agents should be able to perform control and estimation tasks based solely on relative measurements to neighboring agents.

Thus, in the context of aerial robotics, to cooperatively operate multiple vehicles in a GPS-denied environment, only a few of the agents need to localize and navigate relative to the global frame, while the rest of the agents can localize and navigate relative to each other. In the simplest case of two agents, this formulation reduces to a leader-follower scenario, where the leader robot navigates globally and the following robot navigates relative to the leader. The primary advantages of this framework lie in the reduced com- 
plexity, both computational and sensing, of the state estimation for the following robots. By relying on the global information available to the leader robots, the following robots are able to navigate fully by solving the potentially simpler problem of estimating their state relative to the state of the leaders. Also, by estimating state information relative to a known marker, the following robots can be significantly smaller and lighter because external sensors such as laser scanners or stereo cameras are not needed.

The work presented in this paper describes a fast and lightweight sensing solution for estimating the state of a vehicle relative to one of its neighbors. The key contributions of the work are the experimental implementation and validation of the proposed relative sensing solution for generating accurate and high-rate range and bearing estimates relative to a known marker. The framework utilizes an infrared (IR) camera from a commercially available Nintendo Wii ${ }^{1}$ controller and is implemented on an embedded computer. The sensors and embedded computer weigh just over 20 grams. State estimates are available to the controller at $1 \mathrm{kHz}$, with position correction updates coming at $100 \mathrm{~Hz}$, enabling tight position and attitude control. The sensing solution allows for small, lightweight quadrotors with minimal sensing to fly autonomously relative to other vehicles.

The remainder of the paper is organized as follows. Section II summarizes the related work in the field. Sections III and IV detail the sensors used and the integration of the estimation framework. A comparison of the proposed sensing scheme to data collected with a motion capture system is presented in Section V. Finally some conclusions and areas of future work are described in Section VI.

\section{RELATED WORK}

Impressive recent results in the area of quadrotor control have been achieved utilizing statically mounted motion capture systems [6]-[8]. The motion capture systems allow the researcher to focus efforts on controller design and implementation without worrying about state estimation. In addition to the highly accurate measurements available from such systems, tight control is possible in part due to the high rate at which new measurements are taken (typically between 100

\footnotetext{
${ }^{1}$ http://www.nintendo.com/wii
}

and $200 \mathrm{~Hz}$ ) and the low latency with which they are available to the control algorithms.

Using laser scanners as the primary sensor, researchers have demonstrated robust indoor navigation in unstructured environments [1] and transitions between indoor and outdoor flight [2]. Autonomous flight has also been robustly achieved using cameras for position and velocity estimation [3]. Typically, these vehicles are forced to fly slower and more conservatively than those in a motion capture facility since they must do significant on-board computation to generate valid state estimates, thereby limiting the rate at which global position and velocity solutions are available. A notable exception to this convention of slow and conservative flight was created by utilizing information gathered about the environment a priori [9].

Very recent results have highlighted the benefits of utilizing sensors with fast update rates. In [10], the authors propose using a camera with a $100 \mathrm{~Hz}$ update rate and are able to generate state estimates relative to a vertical pole. The work presented in this paper is focused on a cooperative scenario with active markers and so is able to achieve similar state estimate performance at a fraction of the size and computational complexity.

Several papers have proposed new sensing techniques for relative navigation of both ground and aerial robots. Most of the solutions proposed in the literature rely on either time of flight measurements or measuring the received signal strength of a transmitting sensor. One approach proposed sensing neighboring vehicles through generated magnetic fields, thus avoiding the problem of needing good state estimates of the other agents [11]. A good overview of the recent developments in the field is presented in [12] and the associated references, two of which are mentioned here for completeness.

In [12], a 3-D relative sensing solution is proposed consisting of a ring of IR LED transmitters and receivers on two (or more) quadrotors. While this solution generates a full 3-D relative position measurement and is not limited by camera view angles, it requires many LEDs and receivers in a circular pattern, leading to a relatively heavy design (more than 245 grams).

Similar to the idea proposed in this paper, the relative sensing solution proposed in [13] uses a camera pointed at a set of fixed markers to gather relative state information. However, the work presented here 
differs by using a camera with integrated processing and IR LEDs, thus avoiding any image processing and associated computation. Also, in this work, the range and bearing measurements from the IR camera are fused together with IMU data, creating more robust measurements. The IR camera and corresponding data fusion create state estimates at $1 \mathrm{kHz}$ with position corrections at $100 \mathrm{~Hz}$, significantly faster than typical camera refresh rates.

Finally, previous work has demonstrated successful flight using a similar Wii IR camera and active IR LED markers on a quadrotor $[14,15]$. The work in this paper builds on these results by combining the relative position measurements from the camera with on-board inertial sensors to create high-rate filtered data. The filter creates a more robust solution since false range and bearing measurements by the camera will be compensated for by using the inertial sensors. Additionally, in this work the IR LEDs are arranged to allow omindirectional viewing of the marker, giving the following vehicle the ability to fly around the marker.

\section{SEnsing RANGE AND BEARING}

Measuring range and bearing relative to a fixed marker is accomplished by utilizing the IR camera from a Nintendo Wii remote. The Wii functions by extracting the locations of two sets of IR LEDs located in the Nintendo sensor bar. Knowing that the LEDs have a fixed baseline, the Wii remote can calculate range and roll angle relative to the sensor bar $[12,16]$. Similarly, the sensing technique proposed here utilizes a set of active IR LEDs in a known configuration with the IR camera from a Wii remote to detect the IR points $[14,15]$.

Using the camera from a Wii remote offers several distinct advantages over similar methods using vision to detect relatively distinct features $[10,13]$. Most significantly, the Wii IR camera, manufactured by PixArt Imaging, ${ }^{2}$ is completely self-contained, including processing. The camera detects and returns the sizes and pixel centroid locations of the four brightest IR points in the image frame with a $100 \mathrm{~Hz}$ refresh rate. The fast update rate and self-contained processing leaves the micro-controller free to run control and estimation algorithms without having to allocate resources for image processing. Additionally, the camera is very

\footnotetext{
${ }^{2}$ http://www.pixart.com.tw/index.asp
}

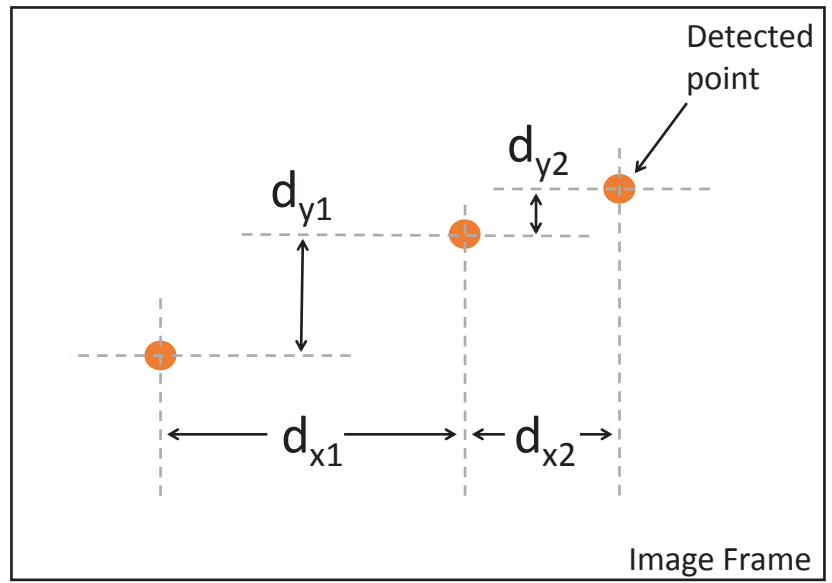

Fig. 2. Definition of distances between detected IR points. The known distances of the points on the marker allow the camera to infer the $x-y$ range, bearing, and $z$ height relative to the markers.

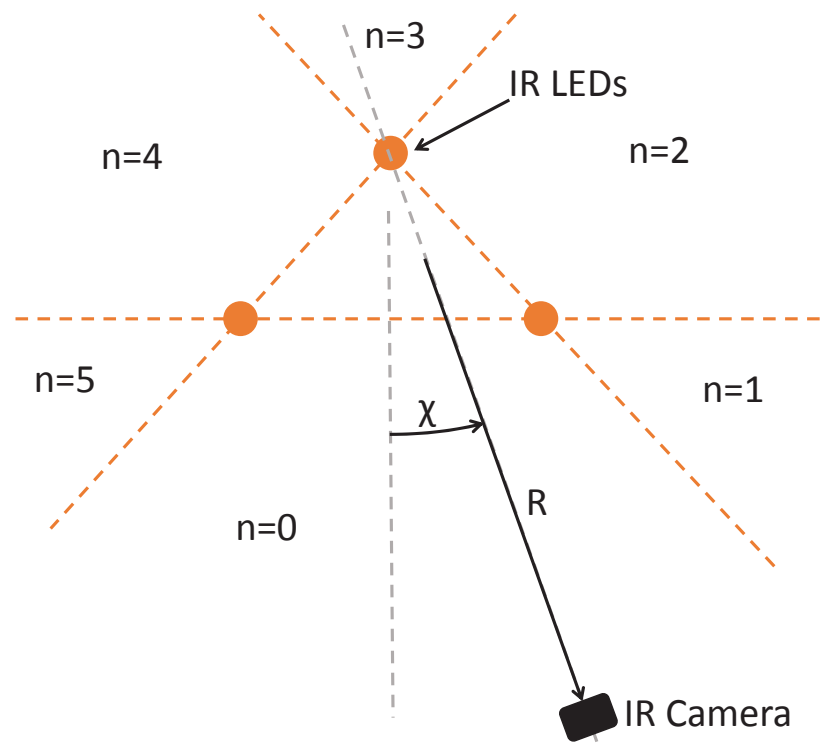

Fig. 3. Top down view of the IR camera looking at the marker. The three LEDs are located at the three points of an equilateral triangle. The three LEDs can be differentiated based on their height, thus partitioning the possible bearing calculation into six regions, $n=0,1, \ldots, 5$.

light weight, weighing only 1.9 grams (including a breakout printed circuit board, oscillator, and connector). The camera and breakout board are shown in Figure 4. Finally, the camera is built, programmed, and optimized specifically to detect IR light sources while ignoring background light, yielding a fairly robust sensor. However, the camera doesn't work well in direct sunlight because of extraneous IR radiation. Also, the field of view of the camera is relatively 


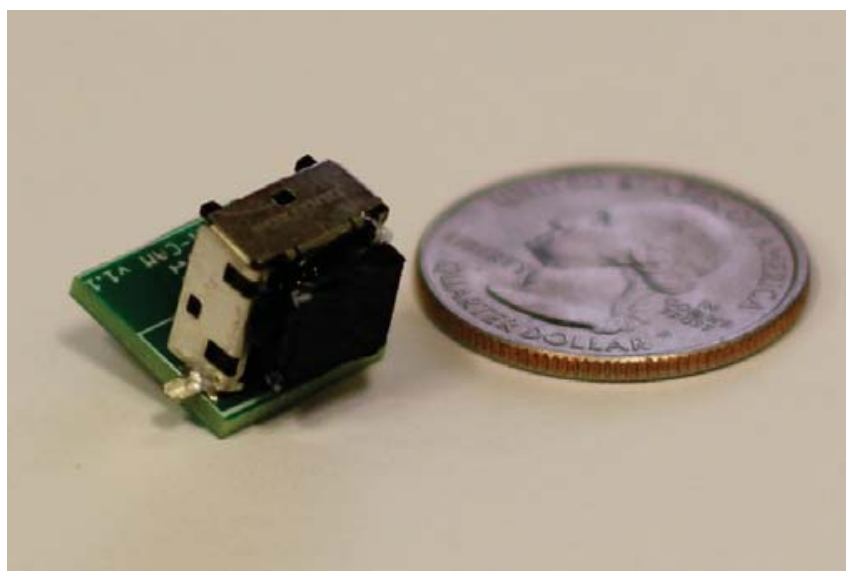

Fig. 4. Infrared camera from a Wii remote mounted on a custom breakout board. The board and connector weigh 1.9 grams. The camera has an IR filter in front to help block out unwanted light.

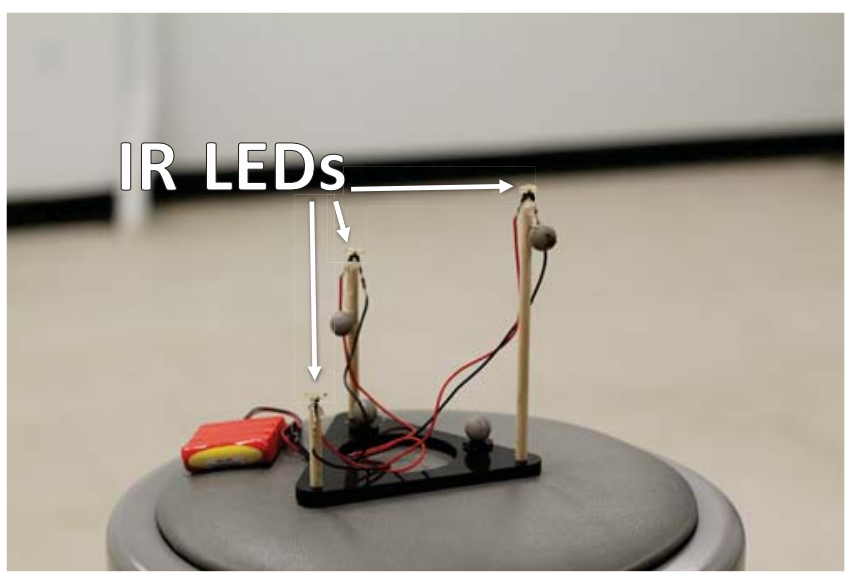

Fig. 5. Marker with three IR LEDs for the IR camera to measure relative range and bearing. The reflective markers are for collecting truth data via the motion capture system.

narrow, about 33 degrees horizontally and 23 degrees vertically ${ }^{3}$. Characterizing the full operating regime of the camera is an area of future research.

The fixed marker used in this work extends the concept of the traditional sensor bar by utilizing three points of IR light instead of just two. An example marker is shown in Figure 5 where the IR LEDs are in a ring around the top of each of the poles so the beam will be visible from all angles. The marker is divided into six regions based on the order in which the three LEDs appear. The identity of the three LEDs is uniquely determined by the camera based on the relative height of each of the LEDs. A top-down view of the markers is shown in Figure 3, including the

\footnotetext{
${ }^{3}$ http://wiibrew.org/wiki/Wiimote
}

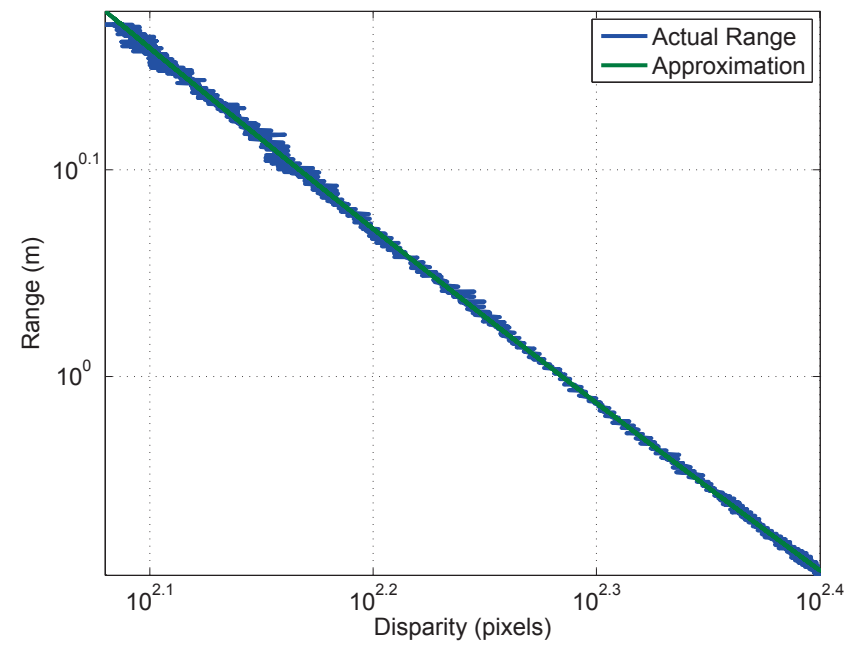

Fig. 6. Measured disparity between outer-most LEDs as a function of range. The approximate data shows the second order least squares fit to the truth data.

six distinct regions. Throughout this paper the marker is assumed to be level. Future work will investigate mounting the marker on a rotating body, such as another quadrotor.

The camera detects the three IR light points and returns the centroid location of each as displayed in Figure 2. From the centroids of the three LEDs, the bearing is calculated by comparing the relative distances of the middle LED to the outer two LEDs. When $d_{x 1}=d_{x 2}$ the camera must be looking straight at the marker. The bearing in each of the marker's regions can thereby be uniquely determined up to \pm 30 degrees. The bearing calculations for each region and for the total bearing, $\chi$, are

$$
\begin{aligned}
\chi_{n} & =(-1)^{n} \tan ^{-1}\left[\left(\frac{2 d_{x 1}}{d_{x 1}+d_{x 2}}-1\right) / \sqrt{3}\right] \\
\chi & =\chi_{n}+n \frac{\pi}{3} .
\end{aligned}
$$

The range to the marker is calculated by looking at the disparity in the $\mathrm{x}$ axis between the two outer-most LEDs. With the range fixed, the disparity value will decrease as the bearing shifts away from center in each region. Thus, the effective disparity, $D_{x}$, is calculated and used based on the bearing angle.

$$
D_{x}=\frac{d_{x 1}+d_{x 2}}{\cos \left(\chi_{n}\right)}
$$

The range is then calculated by fitting a second order polynomial to test data in log-log space. An example 


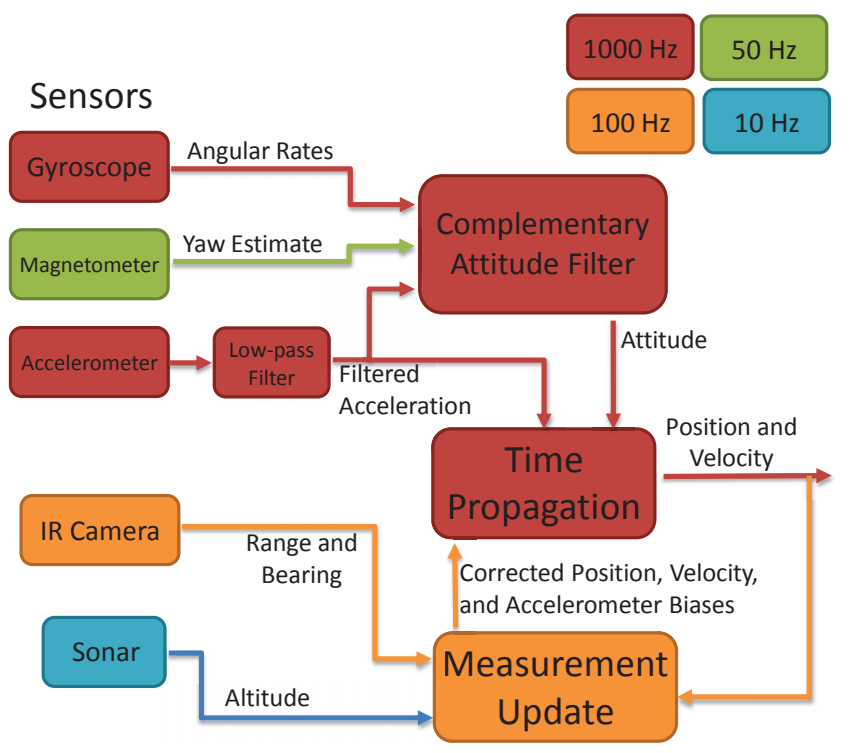

Fig. 7. High level overview of estimation framework developed. Blocks are color coded to denote the rate at which information is being sensed and processed.

data set of disparity versus range with the corresponding polynomial fit is shown in Figure 6. This shows that the relationship is roughly linear in log-log space. The coefficients, $r_{1}, r_{2}$, and $r_{3}$, are found to minimize the fit to the test data in a least squares sense. Using the coefficients, the range is calculated as

$$
R=e^{\left(r_{1}\left(\log D_{x}\right)^{2}+r_{2}\left(\log D_{x}\right)+r_{3}\right)} .
$$

The maximum measurable range of the camera is limited by the brightness of the LEDs, the ability of the camera to detect them, and by the spacing of the LEDs on the marker. Tests are currently in progress to determine the maximum usable range of the marker and camera, primarily by exploring different IR LEDs.

\section{ESTIMATION FRAMEWORK}

The range and bearing measurements calculated by the IR camera are fused together with measurements from inertial sensors, a compass, and a downwardfacing ultrasonic sonar. Similar to the estimation scheme proposed in [2], the sensors are fused together using a sigma point Kalman filter (SPKF). In particular, a square-root central difference Kalman filter is implemented on a micro-controller using the algorithm detailed in [17]. The SPKF integrates forward the acceleration measurements, using the range, bearing, and altitude measurements to estimate the hidden accelerometer biases.
TABLE I. COMPUTATION TIME FOR VARIOUS COMPONENTS OF THE ESTIMATION AND CONTROL ARCHITECTURE.

\begin{tabular}{ll}
\hline Process & $\begin{array}{l}\text { Computation } \\
\text { Time }(\mu \mathbf{s})\end{array}$ \\
\hline Attitude Filter & 18 \\
Time Propagation & 820 \\
XY Measurement Update & 940 \\
Z Measurement Update & 810 \\
Attitude Controller & 2 \\
Position controller & 51 \\
\hline
\end{tabular}

Note that the range and bearing measurements from the IR camera represent a relative measure of position between the camera and the markers. When the markers are stationary, these relative measurements can be directly fused with the on-board inertial measurements. However, when the marker is moving, the relative position measurements can no longer be directly combined with inertial sensors on-board. Provided a communication link between the leader (with the markers) and the follower (with the camera) and provided the leader has a measure of its uncertainty of its state estimates, the follower can combine the state estimates of the leader with the relative position estimates. In particular, by adding the communicated position estimates of the leader to the relative position estimates from the follower in $x$ and $y$ as

$$
\begin{aligned}
& x_{\text {meas }}=x_{\text {rel }}+\hat{x}_{\text {leader }} \\
& y_{\text {meas }}=y_{\text {rel }}+\hat{y}_{\text {leader }}
\end{aligned}
$$

the follower can directly use $x_{\text {meas }}$ and $y_{\text {meas }}$ in its filter. Additionally, as detailed in [18], the uncertainty in these position estimates can be accurately accounted for by adding the component of the covariance matrix of the leader that lies in the direction of the camera sensor to the uncertainty of the relative measurements.

A high level overview of the estimation framework is given in Figure 7 with the color of the various blocks denoting the rate at which the sensor is sampled or the computation is performed. One of the key strengths of the filter is the high rate at which data is processed and available to the micro-controller for use in the control loops. This allows the controller to quickly respond to disturbances, yielding robust, smooth flight [19].

The attitude estimates and gyro biases are calculated independently from the SPKF, using a complementary filter. The gyro measurements are integrated at $1 \mathrm{kHz}$ 

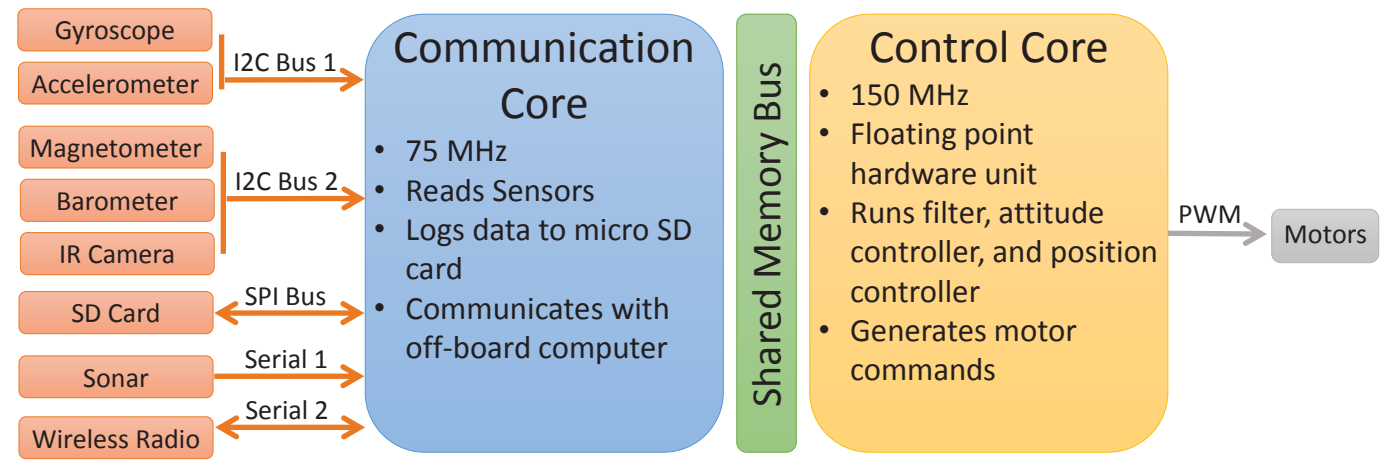

Fig. 8. General computation model used. The processor is divided into two cores. The communication core primarily handles sensor reads and data logging while the control core runs the filter and control algorithms.

and the biases are estimated and the integrated values corrected at $50 \mathrm{~Hz}$ using the accelerometer measurements. The correction step is preformed as detailed in [20].

Keeping the attitude estimates separate from the position and velocity SPKF is done primarily for computational tractability. Estimating the attitude and gyro biases in the SPKF framework (as demonstrated in [17]) is too computationally expensive to be performed quickly on our embedded computer. Also, complementary filters have been shown to work well for estimating attitude, even when operating in an accelerating reference frame, such as on a quadrotor [21]. Future work will investigate the extent to which the attitude estimates can be improved by using an improved model of accelerometer measurements on a quadrotor.

As the full details of the SPKF equations are given in [17], only the details of the time propagation step and measurement model are given here. The time propagation step uses a simple kinematic model of the quadrotor to propagate the estimated states forward based on the current accelerometer readings. The total estimated state is given by $\mathbf{S}=\left[\begin{array}{lll}\mathbf{x}^{T} & \dot{\mathbf{x}}^{T} & \ddot{\mathbf{x}}_{\text {bias }}^{T}\end{array}\right]^{T}$, where each of the elements of $\mathbf{S}$ is a three by one vector containing the $x, y$, and $z$ components of the position, velocity, and accelerometer biases, respectively. Given the current estimates of the state $\mathbf{S}$ at time $i$, the current accelerometer readings $\ddot{\mathbf{x}}_{\text {accel }}$, and the time

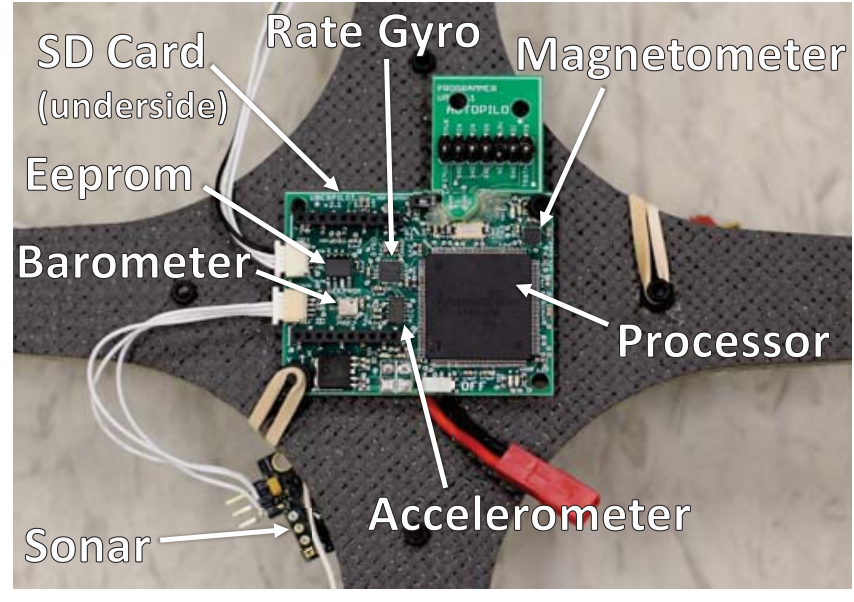

Fig. 9. Autopilot designed for this project. The processor contains two cores and handles all the communication, control, and estimation. Note that the barometer and magnetometer are not used for the results in this paper, but are currently being integrated into the estimation framework.

step $\Delta t$, the updated states are computed as

$$
\begin{aligned}
\mathbf{x}[i+1] & =\mathbf{x}[i]+\Delta t \dot{\mathbf{x}}[i] \\
\ddot{\mathbf{x}}[i] & =\ddot{\mathbf{x}}_{\text {accel }}[i]-\ddot{\mathbf{x}}_{\text {bias }}[i]-\boldsymbol{\eta}_{\text {accel }} \\
\dot{\mathbf{x}}[i+1] & =\dot{\mathbf{x}}[i]+\Delta t\left(\mathbf{R} \ddot{\mathbf{x}}[i]-\left[\begin{array}{lll}
0 & 0 & g
\end{array}\right]^{T}\right) \\
\ddot{\mathbf{x}}_{\text {bias }}[i+1] & =\ddot{\mathbf{x}}_{\text {bias }}[i],
\end{aligned}
$$

where $\mathbf{R}$ is the current rotation matrix from the body frame to the global frame and $\boldsymbol{\eta}_{\text {accel }}$ is additive Gaussian noise. Note that the accelerometer biases are assumed constant. Modelling the biases as evolving according to a random walk shows faster convergence to the true biases [17], but this has not yet been implemented on the embedded system.

The measurement updates are calculated by map- 
ping the range and bearing estimates into Cartesian coordinates, with the filter calculating all estimates and uncertainty values in Cartesian coordinates instead of polar coordinates, thus allowing the linear acceleration measurements to be incorporated with the range and bearing measurements from the IR camera. The measurement model is therefore given by

$$
\mathbf{z}=\left[\begin{array}{c}
-R \cos \chi \\
-R \sin \chi \\
z_{\text {sonar }}
\end{array}\right]+\boldsymbol{\eta}_{\text {position }}
$$

where $\boldsymbol{\eta}_{\text {position }}$ is additive Gaussian noise. Future work will investigate modeling the noise in polar coordinates as described [22].

The estimation and control code is run on a single Texas Instruments F28M35H52C micro-controller ${ }^{4}$. An image of the autopilot designed around this processor is shown in Figure 9. The micro-controller has two separate cores, one dedicated to communication, and one for estimation and control. The separate cores simplify the implementation of the SPKF since the control core can focus completely on running the filter without having to process communication data. The communication core handles all of the sensor reads, communication with an off-board computer via a wireless serial link, and data logging to a micro $\mathrm{SD}$ card. Data is transferred between the cores using a shared memory bus. The control core receives the sensor measurements, runs the attitude filter, SPKF, and control laws, and generates motor commands sent to the four motor controllers. The general processor outline is shown in Figure 8. With the control core running at $150 \mathrm{MHz}$, the processing time for the various computational blocks is given in Table I, showing that none of the individual computation routines requires more than one millisecond.

The control laws implemented on the quadrotor are described in [8] and consist of an attitude controller and a position controller. Position and velocity commands are sent to the quadrotor from an offboard computer. They are then mapped into a desired attitude, from which motor commands are generated.

\section{RESULTS}

The results in this section were collected using the quadrotor in Figure 1. The reflective markers on the vehicle enable the motion capture system to collect

\footnotetext{
${ }^{4}$ http://www.ti.com/product/f28m35h52c
}
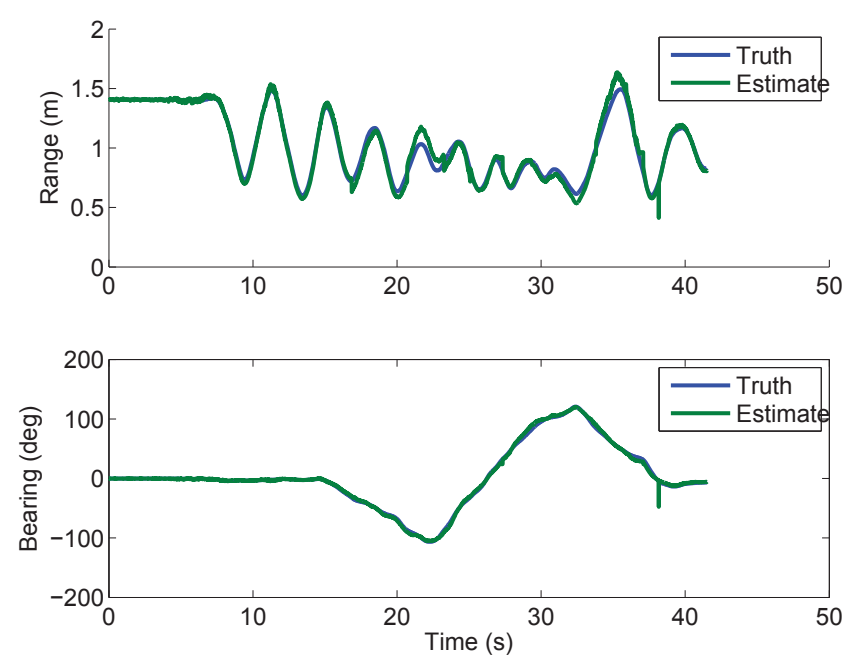

Fig. 10. Range and bearing estimates calculated using only the IR camera (without the SPKF fusing data with the IMU). This data set was collected by moving the camera around by hand. The truth data is collected using a motion capture system with submillimeter accuracy.
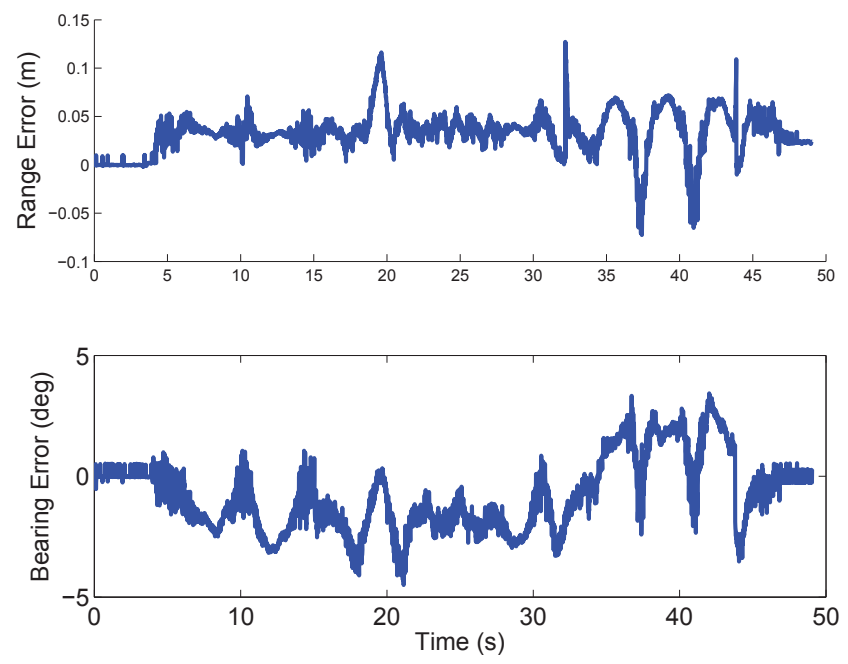

Fig. 11. Error in the range and bearing estimates from just the IR camera.

truth data for the flights. The vehicle and autopilot were designed at the Aerospace Controls Lab at MIT. The autopilot weighs 14.2 grams, the sonar weighs 4.4 grams, and, as mentioned in Section III, the IR camera weighs 1.9 grams. Thus the sensing and computation for the total relative navigation framework weighs just over 20 grams. Initial tests show power consumption of about $170 \mathrm{~mA}$ when running the full filter and about $300 \mathrm{~mA}$ when broadcasting state information over the wireless radio. 

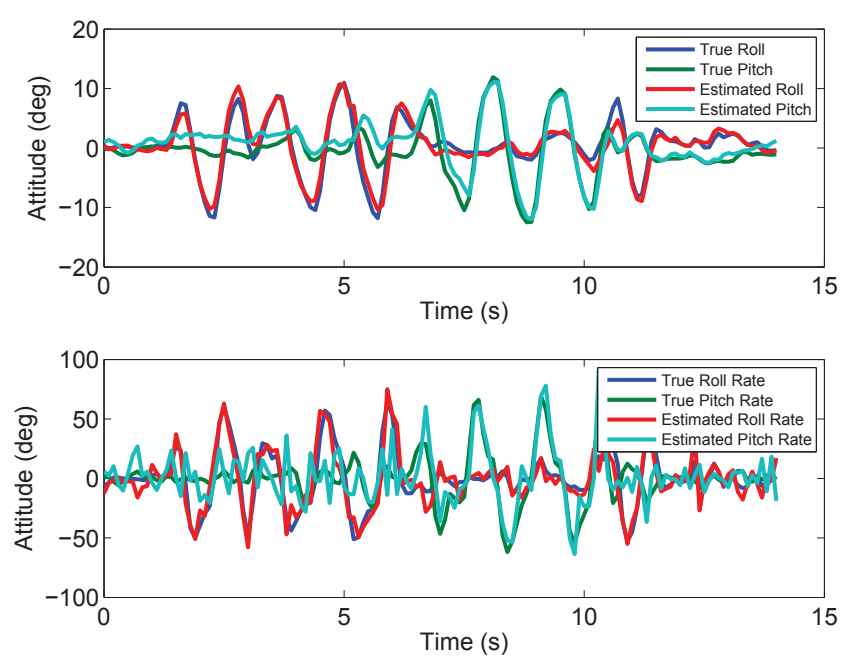

Fig. 12. Comparison of on-board attitude and attitude rate estimates to truth data. The quadrotor is flying in closed-loop attitude control.
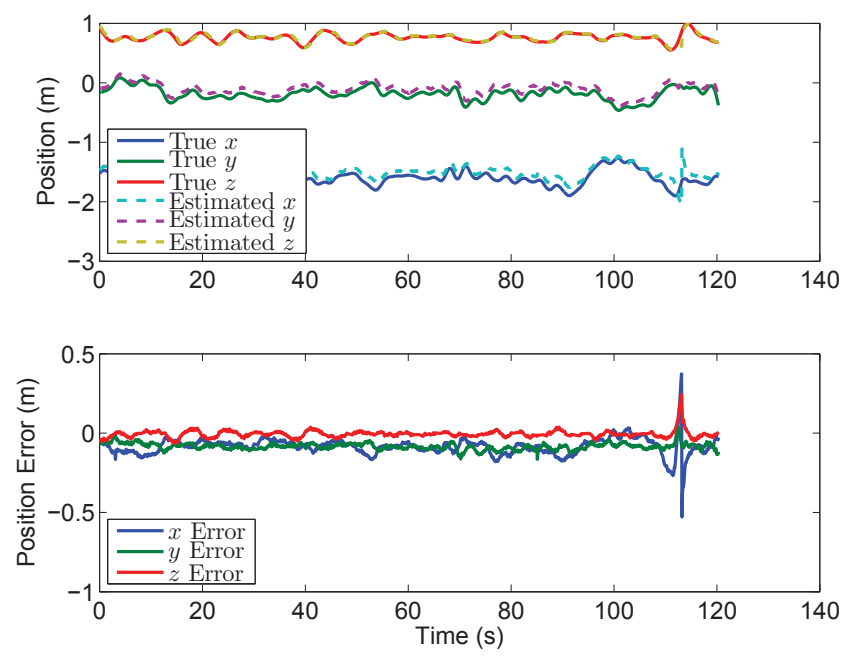

Fig. 13. Comparison of position estimates from the SPKF to truth data during a test flight. The quadrotor is closing the loop around the estimated position and velocities in this flight. Note the spike in estimation error corresponds to the IR camera briefly loosing the IR markers; however, once the markers are found again, the closed-loop controller is able to recover.

The first set of results in Figures 10 and 11 show the performance of the IR camera estimating range and bearing to the marker, independent of the SPKF. This example data set showed a root means square (RMS) error of $4.65 \mathrm{~cm}$ in range and 2.43 degrees in bearing. These data were collected by moving the quadrotor around the marker by hand.

Next, Figure 12 shows the output of the attitude complimentary filter compared to truth data. This data set was captured while the quadrotor was flying with the attitude loop closed. Figure 13 contains position data from another test flight. The position of the vehicle was controlled using the estimated data, thus closing the loop around the state estimates. However, in these particular results, the attitude update (gyro drift correction) information for the complimentary filter is coming from the motion capture system. This will soon be replaced by using the on-board magnetometer for yaw correction.

These results show the initial feasibility of the lightweight sensing scheme for relative navigation. Further work will increase the robustness of the filter and more fully characterize the sensing technique developed.

\section{CONCLUSIONS}

This work details a sensing scheme for obtaining fast and accurate state information relative to a known marker. By utilizing a Wii IR camera, the estimator is able to quickly and reliably detect the location of the IR LEDs on the marker without requiring addition external processing. An on-board estimator combines the camera information with inertial sensors and returns attitude, attitude rate, position, and velocity information for use by a controller at $1 \mathrm{kHz}$. The system is light weight, with the autopilot and sensors weighing just over 20 grams.

There are many areas of future work. First, the attitude yaw loop will be closed on-board using the magnetometer, making the autopilot completely selfcontained. Further work will then investigate modifying the estimation framework to allow for a moving marker. With the marker mounted on another vehicle, the estimator will need to account for both inertial movements and movements relative to the marker. Finally, the estimation scheme will be extended to handle multiple vehicles flying relative to each other.

\section{ACKNOWLEDGMENTS}

This paper is based upon work supported by the National Science Foundation Graduate Research Fellowship under Grant No. 0645960. The authors also acknowledge Boeing Research \& Technology for support of the RAVEN [23] indoor flight facility in which the flight experiments were conducted. 


\section{REFERENCES}

[1] A. Bachrach, S. Prentice, R. He, and N. Roy, "Rangerobust autonomous navigation in gps-denied environments," Journal of Field Robotics, vol. 28, no. 5, pp. 644-666, 2011.

[2] S. Shen and N. Michael, "State estimation for indoor and outdoor operation with a micro-aerial vehicle," in Proc. International Symposium of Robotics Research, IEEE, 2012.

[3] S. Weiss, M. W. Achtelik, S. Lynen, M. Chli, and R. Siegwart, "Real-time onboard visual-inertial state estimation and self-calibration of mavs in unknown environments," in IEEE International Conference on Robotics and Automation (ICRA), pp. 957-964, IEEE, 2012.

[4] S. Shen, N. Michael, and V. Kumar, "Autonomous multifloor indoor navigation with a computationally constrained mav," in Robotics and automation (ICRA), 2011 IEEE international conference on, pp. 20-25, IEEE, 2011.

[5] M. Dorigo, D. Floreano, L. Gambardella, F. Mondada, S. Nolfi, T. Baaboura, M. Birattari, M. Bonani, M. Brambilla, A. Brutschy, et al., "Swarmanoid: a novel concept for the study of heterogeneous robotic swarms," IEEE Robotics \& Automation Magazine, 2012.

[6] S. Lupashin, A. Schollig, M. Sherback, and R. D'Andrea, "A simple learning strategy for high-speed quadrocopter multiflips," in IEEE International Conference on Robotics and Automation (ICRA), pp. 1642-1648, IEEE, 2010.

[7] D. Mellinger and V. Kumar, "Minimum snap trajectory generation and control for quadrotors," in IEEE International Conference on Robotics and Automation (ICRA), 2011.

[8] M. Cutler and J. P. How, "Actuator constrained trajectory generation and control for variable-pitch quadrotors," in AIAA Guidance, Navigation, and Control Conference (GNC), (Minneapolis, Minnesota), August 2012.

[9] A. Bry, A. Bachrach, and N. Roy, "State estimation for aggressive flight in gps-denied environments using onboard sensing," in IEEE International Conference on Robotics and Automation (ICRA), pp. 1-8, May 2012.

[10] I. Sa and P. Corke, " $100 \mathrm{hz}$ onboard vision for quadrotor state estimation," 2012.

[11] K. Sigurd and J. P. How, "UAV Trajectory Design Using Total Field Collision Avoidance," in AIAA Guidance, Navigation, and Control Conference (GNC), (Austin, TX), August 2003 (AIAA-2003-5728).

[12] J. F. Roberts, T. Stirling, J.-C. Zufferey, and D. Floreano, "3-d relative positioning sensor for indoor flying robots," Autonomous Robots, pp. 1-16, 2012.

[13] A. Breitenmoser, L. Kneip, and R. Siegwart, "A monocular vision-based system for $6 \mathrm{~d}$ relative robot localization," in IEEE/RSJ International Conference on Intelligent Robots and Systems (IROS), pp. 79-85, IEEE, 2011.

[14] K. E. Wenzel and A. Zell, "Automatic Take Off, Hovering and Landing Control for Miniature Helicopters with LowCost Onboard Hardware," in Autonome Mobile Systeme 2009, (Karlsruhe, Germany), pp. 73-80, KIT, December 3-4 2009.

[15] K. E. Wenzel, A. Masselli, and A. Zell, "Visual tracking and following of a quadrocopter by another quadrocopter," in IEEE/RSJ International Conference on Intelligent Robots and Systems (IROS 2012), (Vilamoura, Algarve, Portugal), pp. 1-6, IEEE, October 7-12 2012.

[16] J. C. Lee, "Hacking the nintendo wii remote," Pervasive Computing, IEEE, vol. 7, no. 3, pp. 39-45, 2008.

[17] R. Van Der Merwe and E. A. Wan, "Sigma-point kalman filters for integrated navigation," in Proceedings of the 60th Annual Meeting of the Institute of Navigation (ION), pp. 641-654, 2004.

[18] M. Mandic, L. Breger, and J. P. How, "Analysis of decentralized estimation filters for formation flying spacecraft," in AIAA Guidance, Navigation, and Control Conference (GNC), (Providence, RI), August 2004 (AIAA-2004-5135).

[19] D. Gurdan, J. Stumpf, M. Achtelik, K. M. Doth, G. Hirzinger, and D. Rus, "Energy-efficient autonomous four-rotor flying robot controlled at $1 \mathrm{khz}$," in IEEE International Conference on Robotics and Automation (ICRA), pp. 361-366, 2007.

[20] E. Bekir, Introduction to Modern Navigation Systems. World Scientific Publishing, 2007.

[21] C. Chamberlain, "System identification , state estimation, and control of unmanned aerial robots," Master's thesis, Brigham Young University, 2011.

[22] D. Lerro and Y. Bar-Shalom, "Tracking with debiased consistent converted measurements versus ekf," Aerospace and Electronic Systems, IEEE Transactions on, vol. 29, no. 3, pp. 1015-1022, 1993.

[23] J. P. How, B. Bethke, A. Frank, D. Dale, and J. Vian, "Realtime indoor autonomous vehicle test environment," IEEE Control Systems Magazine, vol. 28, pp. 51-64, April 2008. 